Proyecciones Journal of Mathematics Vol. 38, No 2, pp. 221-235, June 2019.

Universidad Católica del Norte

Antofagasta - Chile

\title{
A sine type functional equation on a topological group
}

\author{
D. Zeglami \\ Moulay Ismail University, Morocco \\ S. Kabbaj \\ Ibn Tofail University, Morocco \\ and \\ M. Tial \\ IBn Tofail University, Morocco \\ Received : April 201\%. Accepted: March 2019
}

\begin{abstract}
In [13] H. Stetkcr obtained the complex valued solutions of the functional equation

$$
f\left(x y z_{0}\right) f\left(x y^{-1} z_{0}\right)=f(x)^{2}-f(y)^{2}, \quad x, y \in G,
$$

where $G$ is a topological group and $z_{0} \in Z(G)$ (the center of $G$ ). Our main goal is first to remove this restriction and second, when $G$ is 2divisible and abelian, we will investigate the superstability of the above functional equation.
\end{abstract}

Subjclass. Primary 39B22, 39B32, 39B52.

Keywords. Sine functional equation, character, additive map, superstability. 


\section{Introduction}

Let $G$ be a group. By the sine functional equation on $G$ we will here understand the functional equation

$$
f(x y) f\left(x y^{-1}\right)=f(x)^{2}-f(y)^{2}, \quad x, y \in G,
$$

where $f: G \rightarrow \mathbf{C}$ is the unknown function. Some information, applications and numerous references concerning (1.1) and its further generalizations can be found e.g. in $[1,5-8,10,12-15,19,20]$.

The sine functional equation (1.1) has been generalized by Stetkær in [13]. He determined (under some assumptions on $G$ ) the solutions $f: G \rightarrow$ C of the functional equation

$$
f\left(x y z_{0}\right) f\left(x y^{-1} z_{0}\right)=f(x)^{2}-f(y)^{2}, \quad x, y \in G,
$$

where $z_{0} \in Z(G)$ (the center of $G$ ) [13, Theorem 15.12].

One of the main goal of this paper is to make an advance in the development of the equation (1.2). Chapter 15 of the monograph [13] by Stetkær has been an inspiration by its treatment of the sine functional equation on groups and its results are indispensable for the present work.

The purpose of this paper is about the two main themes:

1. First in Section 3, we extend, in our set up, Stetkær's result about solution of the generalized sine functional equation (1.2), precisely we remove the restriction that $z_{0} \in Z(G)$. An illustrative example on a non-abelian group is presented.

2. In section 4 , we treat a superstability of the integral functional equation $\int_{G} \int_{G}(f(x+y+t) f(x-y+s)) d \mu(t) d \mu(s)=f(x)^{2}-f(y)^{2}, \quad x, y \in$ $G$,

where $G$ is a locally compact and abelian group and $\mu$ is a regular, compactly supported, complex-valued Borel measure on $G$. As second main result we will prove, in the case where $G$ is a 2 -divisible and abelian group, that the functional equation (1.3) is superstable in the class of functions $f: G \longrightarrow \mathbf{C}$, i.e. every such function satisfying the inequality

$$
\left|f\left(x+y+z_{0}\right) f\left(x-y+z_{0}\right)-f(x)^{2}+f(y)^{2}\right| \leq \varepsilon \text { for all } x, y \in G,
$$


where $\varepsilon$ is a fixed positive real number either is bounded or satisfies the functional equation

$$
f\left(x+y+z_{0}\right) f\left(x-y+z_{0}\right)=f(x)^{2}-f(y)^{2},
$$

for all $x, y \in G$. The first results of this kind have been established in [4] for the sine equation, in [2] for the exponential equation, in [3] for the cosine equation on an abelian group and in $[11,16,17,18]$ for generalized Wilson's functional equations on any group.

\section{Notation and terminology}

Let $G$ be a group with neutral element $e, \mathbf{C}$ the field of complex numbers, $\mathbf{R}$ the field of real numbers and $\mathbf{N}$ the set of positive integers. The commutator between $x \in G$ and $y \in G$ is $[x, y]=x y x^{-1} y^{-1}$. Let $[G, G]$ denote the smallest subgroup of $G$ containing the set $\{[x, y] \mid x \in G, y \in G\} .[G, G]$ is called the derived subgroup of $G$.

A function $A: G \rightarrow \mathbf{C}$ is called additive, if it satisfies $A(x y)=A(x)+$ $A(y)$ for all $x, y \in G$.

A character $\chi$ of $G$ is a homomorphism $\chi: G \rightarrow \mathbf{C}^{*}$, where $\mathbf{C}^{*}$ denotes the multiplicative group of non-zero complex numbers. So characters need not be unitary in the present paper. It is well known that the set of characters on $G$ is a linearly independent subset of the vector space of all complex-valued functions on $G$ (see [13, Corollary 3.20]).

For any complex-valued function $f$ on $G$ we use the notation $\check{f}(x)=$ $f\left(x^{-1}\right)$ and $f(x)^{2}=f(x) \times f(x)$ for all $x \in G$.

Let $\delta_{y}, y \in G$ denote the Dirac measure concentrated at $y$.

Let $C(G)$ denote the algebra of all continuous functions from $G$ into $\mathbf{C}$. If $G$ is a locally compact Hausdorff group, then we let $M_{C}(G)$ denote the space of all regular, compactly supported, complex-valued Borel measures on $G$. For $\mu \in M_{C}(G)$, we use the notation $\mu(f)=\int_{G} f(t) d \mu(t)$, for all $f \in C(G)$.

\section{Solution of the generalized sine equation}

In this section we extend, in our set up, Stetkær's result about solution of the functional equation

$$
f\left(x y z_{0}\right) f\left(x y^{-1} z_{0}\right)=f(x)^{2}-f(y)^{2}, \quad x, y \in G,
$$


on groups [13, Theorem 15.12].

The following lemma contains useful properties of the solutions of (3.1).

Lemma 3.1. Let $G$ be a group. If $f$ is a solution of Eq. (3.1) then

(a) The function $g: x \mapsto f\left(x z_{0}\right)$ is a solution of the sine equation (1.1).

(b) $f\left(z_{0}\right)=0$.

Proof. (a) Making the substitution $y=e$ in (3.1) we get $\mathrm{f}^{2}\left(x z_{0}\right)=$ $f^{2}(x)-c, \quad x, y \in G$, where $c:=f(e)^{2}$. So we can reformulate the form of Eq. (3.1) as $\mathrm{f}\left(\mathrm{xyz}_{0}\right) f\left(x y^{-1} z_{0}\right)=f\left(x z_{0}\right)^{2}-f\left(y z_{0}\right)^{2}, \quad x, y \in G$, which means that the function $g: x \mapsto f\left(x z_{0}\right)$ is a solution of the sine equation (1.1).

(b) By puting $x=y=e$ in (3.1) we get that $f\left(z_{0}\right)=0$.

The following theorem relaxes the assumption that $z_{0} \in Z(G)$ of $[13$, Theorem 15.12].

Theorem 3.2. Let $G$ be a group such that $G$ and $[G, G]$ are generated by their squares and let $z_{0}$ be an arbitrarily fixed element in $G$. The solutions $f: G \rightarrow \mathbf{C}$ of (3.1) are the ones of the forms:

(i) $f=c(\chi-\check{\chi})$, where $\chi$ is a character on $G$ such that $\chi\left(z_{0}\right)^{2}=1$ and $c \in \mathbf{C} \backslash\{0\}$ is a constant.

(ii) $f: G \rightarrow \mathbf{C}$ is an additive function such that $f\left(z_{0}\right)=0$.

If $G$ is a topological group and $f$ is continuous then the character from (i) is continuous.

Proof. It is clear that $f \equiv 0$ is a solution of Eq. (3.1), so we suppose that $f \neq 0$. According to the proof of [13, Proposition 15.11] we see that $f(e)=0$. Putting $y=e$ in (3.1) we get that $\mathrm{f}\left(\mathrm{xz}_{0}\right)^{2}=f(x)^{2}, \quad x \in G$, thus

$$
f\left(x z_{0}\right)=\varphi(x) f(x), \quad x \in G,
$$

where $\varphi(x) \in\{ \pm 1\}$. According to Lemma 3.1 the function $x \mapsto f\left(x z_{0}\right)$ is a solution of Eq. (1.1) then from [13, Theorem 15.3] there are only the following two cases:

Case 1: There exist a character $\chi$ of $G$ and $c \in \mathbf{C} \backslash\{0\}$ such that

$$
f\left(x z_{0}\right)=c(\chi(x)-\check{\chi}(x)), \quad x \in G .
$$


Using (3.2) and (3.3), we get that

$$
f(x)=c \varphi(x)(\chi(x)-\check{\chi}(x)), \quad x \in G .
$$

In view of Lemma 3.1 (b) we have $f\left(z_{0}\right)=0$ then using (3.4) we get that $\chi\left(z_{0}\right)=\check{\chi}\left(z_{0}\right)$ i.e. $\chi\left(z_{0}\right)^{2}=1$. Using (3.2), (3.3) and that $\varphi^{2}(x)=1$, we get that

$$
c \varphi\left(x z_{0}\right)\left(\chi\left(x z_{0}\right)-\check{\chi}\left(x z_{0}\right)\right)=c(\chi(x)-\check{\chi}(x)), \quad x \in G .
$$

Substituting $x$ by $x z_{0}^{-1}$ in (3.5) we get that

$$
\varphi(x)(\chi(x)-\check{\chi}(x))=\left(\chi\left(x z_{0}^{-1}\right)-\check{\chi}\left(x z_{0}^{-1}\right)\right), \quad x \in G
$$

which implies, since $\chi\left(z_{0}\right)=\check{\chi}\left(z_{0}\right)$, that

$$
\varphi(x)(\chi(x)-\check{\chi}(x))=\chi\left(z_{0}\right)(\chi(x)-\check{\chi}(x)) \text { for all } \quad x \in G .
$$

Finally, using (3.6) and (3.4), we get

$$
f(x)=c \chi\left(z_{0}\right)(\chi(x)-\check{\chi}(x)) \text { for all } x \in G .
$$

So we arrive at the solution in case $(a)$ with the character $\check{\chi}$ replacing $\chi$ if $\chi\left(z_{0}\right)=-1$.

Case 2: The function $g: x \mapsto f\left(x z_{0}\right)$ is additive. Substituting $x$ by $x z_{0}$ in (3.2) we get the equality

$$
f\left(x z_{0} z_{0}\right)=\varphi\left(x z_{0}\right) f\left(x z_{0}\right), \quad x \in G,
$$

wich implies that

$$
f\left(x z_{0}\right)+f\left(z_{0}^{2}\right)=\varphi\left(x z_{0}\right) f\left(x z_{0}\right), \quad x \in G .
$$

Making the substitutions $x=e$ in (3.7) and using the fact that $f\left(z_{0}\right)=0$ we get $f\left(z_{0}^{2}\right)=0$. Then from (3.8) we obtain that

$$
f(x)=\varphi(x) f(x) \text { for all } x \in G,
$$

which says, by the use of (3.2), that $f(x)=f\left(x z_{0}\right)$ for all $x \in G$. Thus $f$ is additive. So, we are in case $(b)$ of our statement.

Conversely, it is a simple calculation to check that in the two cases listed above the given functions are solutions of the functional equation (3.1). 
Problem 3.3. Let $G$ be a locally compact Hausdorff group such that $G$ and $[G, G]$ are generated by their squares. Let $\mu$ be a regular, compactly supported, complex-valued Borel measures on $G$. Find the form of continuous solutions $f: G \rightarrow \mathbf{C}$ of the sine functional equation on integral form

$$
\int_{G} \int_{G}\left(f(x y t) f\left(x y^{-1} s\right)\right) d \mu(t) d \mu(s)=f(x)^{2}-f(y)^{2}, \quad x, y \in G .
$$

Note that Eq. (3.1) results from (3.9) by taking $\mu=\delta_{z_{0}}$. It's easy to see that if $f$ is a solution of (3.9) then the function $x \mapsto \int_{G} f(x t) d \mu(t)$ is a solution of the sine equation (1.1) and that the functions $f: G \rightarrow \mathbf{C}$ of the forms:

(a) $f=c(\chi-\check{\chi})$, where $\chi$ is a continuous character on $G$ satisfying $\mu(\chi)=\mu(\check{\chi})= \pm 1$, and where $c \in \mathbf{C} \backslash\{0\}$ is a constant,

(b) $f: G \rightarrow \mathbf{C}$ is a non-zero continuous additive function such that $\mu(f)=0$, here it is necessary that $\mu(G)= \pm 1$,

are solutions of (3.9). Conversely, it seems natural to conjecture that any solution of (3.9) is of one of the forms presented in (a) or (b)?

Remark 3.4. Let $z_{0} \in \mathbf{R}^{*}$. For an application of Theorem 3.2 on an abelian group. We find in [13, Corollary 15.13] that the continuous solutions $f: \mathbf{R} \longrightarrow \mathbf{C}$ of the functional equation

$$
f\left(x+y+z_{0}\right) f\left(x-y+z_{0}\right)=f(x)^{2}-f(y)^{2}, \quad x, y \in \mathbf{R},
$$

are the functions of the following form:

$$
f(x)=c \sin \left(\frac{n \pi}{z_{0}} x\right), x \in \mathbf{R},
$$

where $c \in \mathbf{C}$ and $n \in \mathbf{Z}$ are constants.

Example 3.5. For an application of our result on a non-abelian group. Consider the $(a x+b)$-group $G:=\left\{\left(\begin{array}{cc}a & b \\ 0 & 1\end{array}\right) \mid a>0, b \in \mathbf{R}\right\}$. Let $Z_{0}=\left(\begin{array}{cc}a_{0} & b_{0} \\ 0 & 1\end{array}\right)$ be a fixed element on $G$ such that $a_{0} \neq 1$. The assumptions of Theorem 3.2 that $G$ and $[G, G]$ are generated by their squares can be easily verified. We indicate here the corresponding continuous solutions of Eq. (3.1) viz. the generalized sine functional equation 


$$
f\left(X Y Z_{0}\right) f\left(X Y^{-1} Z_{0}\right)=f^{2}(X)-f^{2}(Y), X, Y \in G .
$$

The continuous characters on $G$ are parametrized by $\lambda \in \mathbf{C}$ as follows (see, e.g., [13, Example 3.13]):

$$
\chi_{\lambda}\left(\begin{array}{ll}
a & b \\
0 & 1
\end{array}\right)=a^{\lambda} \text { for } a>0 \text { and } b \in \mathbf{R} .
$$

So the condition $\chi_{\lambda}^{2}\left(Z_{0}\right)=1$ becomes $e^{2 \lambda \ln a_{0}}=1$, which reduces to $\lambda=i \frac{n \pi}{\ln a_{0}}$, where $n \in \mathbf{Z}$. The relevant characters are thus

$$
\chi_{n}\left(\begin{array}{cc}
a & b \\
0 & 1
\end{array}\right):=a^{i \frac{n \pi}{\ln a_{0}}}=e^{i n \pi \frac{\ln a}{\ln a_{0}}} \text { for } a>0, b \in \mathbf{R} \text { and } n \in \mathbf{Z}
$$

and so,

$$
\begin{aligned}
\check{\chi}_{n}\left(\begin{array}{cc}
a & b \\
0 & 1
\end{array}\right) & =\chi_{n}\left(\begin{array}{cc}
a & b \\
0 & 1
\end{array}\right)^{-1}=\chi_{n}\left(\begin{array}{cc}
\frac{1}{a} & \frac{-b}{a} \\
0 & 1
\end{array}\right) \\
& =e^{-i n \pi \frac{\ln a}{\ln a_{0}}} \text { for } a>0, b \in \mathbf{R} \text { and } n \in \mathbf{Z} .
\end{aligned}
$$

In view of [13, Example 2.10] the continuous additive functions on $G$ are parametrized by $\gamma \in \mathbf{C}$ as follows

$$
A_{\gamma}\left(\begin{array}{cc}
a & b \\
0 & 1
\end{array}\right)=\gamma \ln a \text { for } a>0 \text { and } b \in \mathbf{R}
$$

In the point (ii) of Theorem 3.2 we have $A_{\gamma}\left(Z_{0}\right)=0$ which reduces, since $a_{0} \neq 1$, to $\gamma=0$ i.e. $A_{\gamma}=0$. So this case does not occur here.

In conclusion, by help of Theorem 3.2, we find that the continuous solutions $f: G \longrightarrow \mathbf{C}$ of Eq. (3.10) are the functions of the following form:

$$
f_{n}\left(\begin{array}{ll}
a & b \\
0 & 1
\end{array}\right)=c \sin \left(\frac{n \pi}{\ln a_{0}} \ln a\right) \text { for } a>0 \text { and } b \in \mathbf{R},
$$

where $c \in \mathbf{C}$ and $n \in \mathbf{Z}$ are constants. 


\section{Superstability of Eq. (1.2)}

For the proof of our results we will begin by pointing out a superstability result for the equation

$$
g(x+y) g(x-y)=f(x)^{2}-f(y)^{2}, x, y \in G,
$$

where $(G,+)$ is an abelian group and $f, g: G \rightarrow \mathbf{C}$ are the unknown functions.

Proposition 4.1. ([9]) Let $\delta>0$ be given and let $G$ be a 2-divisible, abelian group. Assume that functions $f, g: G \rightarrow \mathbf{C}$ satisfy the inequality

$$
\left|g(x+y) g(x-y)-f(x)^{2}+f(y)^{2}\right| \leq \delta \text { for all } x, y \in G,
$$

and that $g$ is unbounded. Then $g$ satisfies the functional equation

$$
g(x+y) g(x-y)=g(x)^{2}-g(y)^{2}, \quad x, y \in G .
$$

Proof. We refer to [9, Theorem 1] for a proof.

The following lemma contains a useful property of the solutions $f: G \rightarrow$ C of the inequality (4.3).

Lemma 4.2. Let $G$ be an abelian and locally compact group and let $\delta>0$ be given. Assume that the continuous function $f: G \rightarrow \mathbf{C}$ satisfies the inequality

$$
\left|\int_{G} \int_{G}(f(x+y+t) f(x-y+s)) d \mu(t) d \mu(s)-f(x)^{2}+f(y)^{2}\right| \leq \delta
$$

for all $x, y \in G$. Then the function $x \mapsto \int_{G} f(x+t) d \mu(t)$ is unbounded if and only if $f$ is unbounded.

Proof. Letting $y=e$ in (4.3) we get that

$$
\left|\left(\int_{G} f(x+t) d \mu(t)\right)^{2}-f(x)^{2}\right| \leq \delta+\left|f(e)^{2}\right|
$$

which shows that the function $g$, defined by $g(x):=\int_{G} f(x+t) d \mu(t)$ for all $x \in G$, is unbounded iff $f$ is unbounded. 
In Proposition 1 below, we will study a superstability result of the functional equation

$$
\begin{gathered}
\int_{G} \int_{G}(f(x+y+t) f(x-y+s)) d \mu(t) d \mu(s)=f(x)^{2}-f(y)^{2}, \\
x, y \in G
\end{gathered}
$$

on any abelian and locally compact group $(G,+)$.

Proposition 4.3. Let $G$ be a 2-divisible, abelian and locally compact group and let $\delta>0$ be given. Assume that the continuous function $f: G \rightarrow \mathbf{C}$ satisfies the inequality (4.3) for all $x, y \in G$. Then either $f$ is bounded or $f$ satisfies the functional equation

$$
\begin{aligned}
& \int_{G} \int_{G}(f(x+y+t) f(x-y+s)) d \mu(t) d \mu(s) \\
= & \left(\int_{G} f(x+t) d \mu(t)\right)^{2}-\left(\int_{G} f(y+t) d \mu(t)\right)^{2},
\end{aligned}
$$

for all $x, y \in G$.

Proof. Assume that $f$ satisfies the inequality (4.3). Define $g: G \rightarrow \mathbf{C}$ by

$$
g(x)=\int_{G} f(x+t) d \mu(t) \quad \text { for all } \quad x \in G .
$$

Using (4.6), the inequality (4.3) becomes

$$
\left|g(x+y) g(x-y)-f(x)^{2}+f(y)^{2}\right| \leq \delta,
$$

Suppose that $f$ is unbounded, Lemma 4.2 tells us that so is $g$. Then according to Proposition 4.1 we infer that $g$ satisfies the equation

$$
g(x+y) g(x-y)=g(x)^{2}-g(y)^{2},
$$

for all $x, y \in G$. Hence we conclude that either $f$ is bounded or it is a solution of the integral functional equation (4.5).

As consequences of Proposition 4.3 one can obtain the following corollary. 
Corollary 4.4. Let $G$ be a 2 -divisible abelian group, let $z_{0} \in G$, and let $\delta>0$ be given. Assume that the function $f: G \rightarrow \mathbf{C}$ satisfies the inequality

$$
\left|f\left(x+y+z_{0}\right) f\left(x-y+z_{0}\right)-f(x)^{2}+f(y)^{2}\right| \leq \delta,
$$

for all $x, y \in G$. Then either $f$ is bounded or the function $x \mapsto f\left(x+z_{0}\right)$ satisfies the equation (4.2).

Proof. As the proof of Proposition 4.3 with $\mu=\delta_{z_{0}}$.

In Theorem 4.5 below, the superstability of the equation

$$
f\left(x+y+z_{0}\right) f\left(x-y+z_{0}\right)=f(x)^{2}-f(y)^{2}, \quad x, y \in G .
$$

will be investigated on any 2-divisible, abelian group.

Theorem 4.5. Let $G$ be a 2-divisible abelian group, let $z_{0}$ be an arbitrarily fixed element in $G$, and let $\delta>0$ be given. Assume that the function $f: G \rightarrow \mathbf{C}$ satisfies the inequality

$$
\left|f\left(x+y+z_{0}\right) f\left(x-y+z_{0}\right)-f(x)^{2}+f(y)^{2}\right| \leq \delta,
$$

for all $x, y \in G$. Then either $f$ is bounded or it satisfies the equation (4.7).

Proof. Let $f$ be an unbounded function satisfying the inequality (4.8). According to Corollary 4.4, the function $x \mapsto f\left(x+z_{0}\right)$ is a solution of the functional equation (4.2), i.e. we have

(4.9) $f\left(x+y+z_{0}\right) f\left(x-y+z_{0}\right)=f\left(x+z_{0}\right)^{2}-f\left(y+z_{0}\right)^{2}, \quad x, y \in G$,

then $f\left(z_{0}\right)=0$. From the inequality (4.8) combined with (4.9) we get

$$
\left|f\left(x+z_{0}\right)^{2}-f\left(y+z_{0}\right)^{2}-f(x)^{2}+f(y)^{2}\right| \leq \delta, x, y \in G,
$$

which implies, when we put $y=e$, that

$$
\left|f\left(x+z_{0}\right)^{2}-f(x)^{2}\right| \leq \delta^{\prime} \text { for all } x \in G,
$$

where $\delta^{\prime}=\delta+\left|f(e)^{2}\right|$. Equality (4.9) tells us that the function $x \mapsto f\left(x+z_{0}\right)$ is a solution of the functional equation (1.1), then from [13, Corollary 15.5] there are only the following two cases: 
Case 1. The function $x \mapsto f\left(x+z_{0}\right)$ is additive, i.e.

$$
f(x)=a\left(x-z_{0}\right), \quad x \in G,
$$

where $a$ is an unbounded additive function. Using (4.10) we get

$$
\left|a(x)^{2}-a\left(x-z_{0}\right)^{2}\right| \leq \delta^{\prime}, \quad x, y \in G,
$$

then

$$
\left|2 a\left(z_{0}\right) a(x)-a\left(z_{0}\right)^{2}\right| \leq \delta^{\prime}, \quad x, y \in G,
$$

which implies that $a\left(z_{0}\right)=0$ because $a$ is unbounded. So $f(x)=a(x)$ and $a\left(z_{0}\right)=0$. Hence we conclude, using Theorem 3.2, that $f$ is a solution of Eq. (4.7). ( $G$ and $[G, G]$ are generated by their squares because $[G, G]=\{e\}$ and $G$ is 2-divisible).

Case 2. There exist a character $\chi$ of $G$ and $c \in \mathbf{C} \backslash\{0\}$ such that

$$
f\left(x+z_{0}\right)=c(\chi(x)-\check{\chi}(x)), \quad x \in G,
$$

then

$$
f(x)=c\left(\chi\left(x-z_{0}\right)-\check{\chi}\left(x-z_{0}\right)\right), \quad x \in G .
$$

Using (4.10) we get

$$
\left|c^{2}\right|\left|(\chi(x)-\check{\chi}(x))^{2}-\left(\chi\left(x-z_{0}\right)-\check{\chi}\left(x-z_{0}\right)\right)^{2}\right| \leq \delta^{\prime}, \quad x, y \in G,
$$

which means that

$$
\left|c^{2}\right| \mid\left(\chi(x)^{2}+\check{\chi}(x)^{2}-\chi(x)^{2} \check{\chi}\left(z_{0}\right)^{2}-\check{\chi}(x)^{2} \chi\left(z_{0}\right)^{2} \mid \leq \delta^{\prime}, \quad x, y \in G,\right.
$$

then

$$
\left|c^{2}\right|\left|\left(1-\check{\chi}\left(z_{0}\right)^{2}\right) \chi(x)^{2}+\left(1-\chi\left(z_{0}\right)^{2}\right) \check{\chi}(x)^{2}\right| \leq \delta^{\prime}, \quad x, y \in G,
$$

implying, after dividing by $\check{\chi}\left(z_{0}\right)$ and using the equality $1-\check{\chi}\left(z_{0}\right)^{2}=$ $\frac{\chi\left(z_{0}\right)^{2}-1}{\chi\left(z_{0}\right)^{2}}$, that 


$$
\left|c^{2} \frac{\chi\left(z_{0}\right)^{2}-1}{\chi\left(z_{0}\right)}\right|\left|\frac{\chi(x)^{2}}{\chi\left(z_{0}\right)}-\chi\left(z_{0}\right) \check{\chi}(x)^{2}\right| \leq \delta^{\prime}, \quad x, y \in G .
$$

Or $G$ is 2 -divisible then there exists $u_{0} \in G$ such that $z_{0}=2 u_{0}$. So from (4.12) we infer that

$$
\left|c^{2} \frac{\chi\left(z_{0}\right)^{2}-1}{\chi\left(z_{0}\right)}\right|\left|\chi\left(x-u_{0}\right)^{2}-\check{\chi}\left(x-u_{0}\right)^{2}\right| \leq \delta^{\prime}, \quad x, y \in G .
$$

Since $f$ is unbounded then so is the function

$$
x \mapsto f\left(x+z_{0}\right)=c(\chi(x)-\check{\chi}(x)),
$$

this shows that at least one of the characters $\chi$ or $\check{\chi}$ is unbounded (in fact, both are unbounded because $\check{\chi}(x)=\chi(-x), x \in G)$. Then we can choose a sequence $\left\{y_{n}\right\}$ in $G$ such

$$
\left|\chi\left(y_{n}\right)\right| \rightarrow \infty \text { as } n \rightarrow \infty
$$

which implies that

$$
\left|\check{\chi}\left(y_{n}\right)\right| \rightarrow 0 \quad \text { as } n \rightarrow \infty .
$$

Consequently, we get

$$
\lim _{n \rightarrow \infty}\left(\left|\chi\left(y_{n}\right)^{2}\right|-\left|\check{\chi}\left(y_{n}\right)^{2}\right|\right)=\infty \text {. }
$$

Since

$$
\left|\chi\left(y_{n}\right)^{2}\right|-\left|\check{\chi}\left(y_{n}\right)^{2}\right| \leq\left|\chi\left(y_{n}\right)^{2}-\check{\chi}\left(y_{n}\right)^{2}\right| \quad \text { for all } n \in \mathbf{N}
$$

then $\left|\chi\left(y_{n}\right)^{2}-\check{\chi}\left(y_{n}\right)^{2}\right| \rightarrow \infty$ as $n \rightarrow \infty$. Therefore we obtain that $\chi^{2}-\check{\chi}^{2}$ is unbounded and so is the function

$$
x \mapsto \chi\left(x-u_{0}\right)^{2}-\check{\chi}\left(x-u_{0}\right)^{2},
$$

which, in the light of (4.13), tells us that necessarily we have $\chi\left(z_{0}\right)^{2}=1$ and it follows, by the use of (4.11), that

$$
f(x)=c \check{\chi}\left(z_{0}\right)(\chi(x)-\check{\chi}(x)), \quad x \in G .
$$

Hence we conclude via Theorem 3.2 that $f$ is a solution of Eq. (4.7). The proof of the theorem is completed.. 
Let $\mathcal{H}$ be a real Hilbert space and let $z_{0} \in \mathcal{H}$. As consequence of Theorem 4.5, In the following corollary, we give the superstability of the functional equation

$$
f\left(x+y+z_{0}\right) f\left(x-y+z_{0}\right)=f(x)^{2}-f(y)^{2}, x, y \in \mathcal{H},
$$

which was studied in e.g. [10].

Corollary 4.6. Let $\mathcal{H}$ be a real Hilbert space with linear inner product $\langle\cdot, \cdot\rangle$, Let $z_{0} \in \mathcal{H}$, and let $\delta>0$ be given. Assume that the continuous function $f: \mathcal{H} \rightarrow \mathbf{R}$ satisfies the inequality

$$
\left|f\left(x+y+z_{0}\right) f\left(x-y+z_{0}\right)-f(x)^{2}+f(y)^{2}\right| \leq \delta,
$$

for all $x, y \in \mathcal{H}$. Then $f$ is unbounded or $f$ has one of the following three forms where $c \in \mathbf{R}$ and $x_{0} \in \mathcal{H}$ denote constants:

$$
f(x)=c \sinh \left\langle x, x_{0}\right\rangle ; \quad f(x)=c \sin \left\langle x, x_{0}\right\rangle \text { or } f(x)=c\left\langle x, x_{0}\right\rangle,
$$

for all $x \in \mathcal{H}$.

Proof. The proof follows from Theorem 4.5 combined with [13, Exercise $15.8]$.

Acknowledgement. The authors wishe to thank the referee for a number of constructive comments which have led to essential improvement of the paper. We are indebted to Professor Henrik Stetkær for a helpful conversation which contributed to the first part of this article.

\section{References}

[1] J. Aczél, J. Dhombres, Functional Equations in Several Variables, Cambridge University Press, New York, (1989).

[2] J. A. Baker, J. Lawrence, and F. Zorzitto, The stability of the equation $f(x+y)=f(x) f(y)$, Proc. Amer. Math. Soc. 74, pp. 242-246, (1979).

[3] J. A. Baker, The stability of the cosine equation, Proc. Amer. Math. Soc. 80 , pp. 411-416, (1980). 
[4] P. W. Cholewa, The stability of the sine equation, Proc. Amer. Math. Soc. 88, No. 4, pp. 631-634, (1983).

[5] J. Chung and D. Kim, Sine functional equation in several variables, Archiv der Mathematik 86, No 5, pp. 425-429, (2006).

[6] I. Corovei, The sine functional equation on 2-divisible groups, Mathematica 47, No 1, pp. 49-52, (2005).

[7] Pl. Kannappan, On sine functional equation, Studia Sci. Math. Hung., 4, pp. 331-333, (1969).

[8] Pl. Kannappan, Functional Equations and Inequalities with Applications. Springer Monographs in Mathematics. Springer, New York, xxiv +810 , (2009).

[9] G. H. Kim, A stability of the generalized sine functional equations, J. Math. Anal. Appl., 331, pp. 886-894, (2007).

[10] S. Kurepa, On the functional equation $f(x+y) f(x-y)=f(x)^{2}-f(y)^{2}$, Ann. Polon. Math., 10 , pp. 1-5, (1961).

[11] A. Roukbi, D. Zeglami and S. Kabbaj, Hyers-Ulam stability of Wilson's functional equation, J. Math. Sci. Adv. Appl., 22, pp. 19-26, (2013).

[12] P. Sinopoulos, Generalized sine equations, I, Aequationes math., 48, No. 2, pp. 171-193, (1994).

[13] H. Stetkær, Functional equations on groups, World Scientific Publishing, Hackensack, xvi+378, (2013).

[14] H. Stetkær, Van Vleck's functional equation for the sine. Aequationes Math. 90 (1), pp. 25-34, (2016).

[15] E. B. Van Vleck, A functional equation for the sine, Ann. of Math., Second Series, 11 (4), pp. 161-165, (1910).

[16] D. Zeglami, B. Fadli, S. Kabbaj, On a variant of $\mu$-Wilson's functional equation on a locally compact group, Aequationes Math., 89, pp. 12651280, (2015).

[17] D. Zeglami, A. Charifi and S. Kabbaj, Superstability problem for a large class of functional equations, Afr. Mat., 27, pp. 469-484, (2016). 
[18] D. Zeglami, M. Tial and B. Fadli, Wilson's Type Hilbert-space valued functional equations, Adv. Pure. Appl. Math., 7, No. 3, pp. 189-196, (2016).

[19] D. Zeglami and B. Fadli, Integral functional equations on locally compact groups with involution, Aequationes Math., 90 (5), pp. 967-982.

[20] D. Zeglami, M. Tial, S. Kabbaj, The integral sine addition law, Submited to Proyecciones J. of Math.

D. Zeglami

Department of Mathematics,

E. N. S. A. M,

Moulay Ismail University,

B. P: 15290 Al Mansour, Meknes,

Morocco

e-mail : zeglamidriss@yahoo.fr

\section{S. Kabbaj}

Department of Mathematics,

Faculty of Sciences,

Ibn Tofail University,

B. P. : 14000. Kenitra,

Morocco

e-mail : samkabbaj@yahoo.fr

and

\section{Tial}

Department of Mathematics,

Faculty of Sciences,

Ibn Tofail University,

B. P. : 14000. Kenitra,

Morocco

e-mail : tialmohamed@gmail.com 